Research Article

\title{
Preparation and Aromatic Hydrocarbon Removal Performance of Potassium Ferrate
}

\author{
Wei Guan, Zhigang Xie, and Jia Zhang \\ Chongqing Key Laboratory of Environmental Materials \& Remediation Technologies, Chongqing University of Arts and Sciences, \\ Chongqing 402160, China \\ Correspondence should be addressed to Zhigang Xie; xinghu2200@163.com
}

Received 22 July 2014; Accepted 9 August 2014; Published 2 September 2014

Academic Editor: Wen Zeng

Copyright (C) 2014 Wei Guan et al. This is an open access article distributed under the Creative Commons Attribution License, which permits unrestricted use, distribution, and reproduction in any medium, provided the original work is properly cited.

\begin{abstract}
This experiment adopts the hypochlorite oxidation method to constantly synthesize potassium ferrate. Under the condition of micropolluted source water $\mathrm{pH}$ and on the basis of naphthalene, phenanthrene, and pyrene as research objects, the effects of different systems to remove aromatic hydrocarbons were studied. Various oxidation systems to remove phenanthrene intermediate are analyzed and the detailed mechanisms for removal of phenanthrene are discussed. The study found that the main intermediate of potassium ferrate system to transform phenanthrene is 9,10 -phenanthraquinone and its area percentage reached $82.66 \%$; that is, 9,10-phenanthraquinone is the key entity to remove phenanthrene.
\end{abstract}

\section{Introduction}

Ferrate is an ideal water treatment reagent. In acidic medium, ferrate redox potential is very high; in a wide $\mathrm{pH}$ range, its oxidation is strong, and it has strong deactivating effect on microorganisms. Ferrate in sewage purification shows excellent oxidation, adsorption, and flocculation to remove contaminants, and it has stronger sterilization effect than chlorine department oxidant [1-3]. Ferrate is the best oxidant replacement for chlorine source as water purification agent. In addition, ferrate as the positive active material of the green power supply has high electrode potential and capacitance [4-6]. This unique feature makes ferrates environmentally friendly green oxidation reagents with dual function.

The ferrate crystal belongs to orthogonal crystal system. For potassium ferrate $\left(\mathrm{K}_{2} \mathrm{FeO}_{4}\right)$, ferrate has regular tetrahedron structure. $\mathrm{Fe}$ atom is at the center of the tetrahedron, with four oxygen atoms in the four corners of tetrahedron, presenting a slightly distorted tetrahedron structure. Ferrate is a deep purple solid, its solution has specific purple color, and maximum absorption wavelength is $510 \mathrm{~nm}$. At the same time, ferrate ion has an absorption peak at $786 \mathrm{~nm}$, which is also its characteristic absorption peak. The main compound in ferrate is potassium ferrate and in solid state it is dark purple powder, and melting point is $198^{\circ} \mathrm{C}$. When ferrate is in dry conditions, it starts to break down at $230^{\circ} \mathrm{C}$ [7-9].

Iron (VI) ions in potassium ferrate, located in the highest valence state of iron, have strong oxidativity; also they have selectivity. They can oxidize plenty of organics. Their oxidativity is significantly stronger than conventional water treatment oxidant, such as potassium permanganate, ozone, and hypochlorite. Under acid medium their standard electrode potential is $2.20 \mathrm{~V}$; however, it is $0.72 \mathrm{~V}$ in alkaline condition. We can modify the ferrate cations' structure and adjust $\mathrm{pH}$ value to control the oxidation activity, so as to achieve high selectivity [10-14].

Heavy metal pollutants in the micropolluted water, such as cadmium and manganese, can be removed by potassium ferrate, and the removal rate reached $80 \%$. In addition, potassium ferrate has good ability to remove organic compounds, such as phenols, alcohols, phenols, organic acid, organic nitrogen, and amino acid, lipid compounds containing sulfur and benzene, and some refractory compounds.

At present, much domestic and foreign source water is polluted by aromatic hydrocarbon. The concentration of aromatic hydrocarbon in water is very low, but the removal efficiency of conventional water treatment technology is not ideal. Due to the special stability of the benzene ring 
TABLE 1: Main reagents and their specifications.

\begin{tabular}{lccc}
\hline Number & Name & Chemical level & Manufacturer \\
\hline 1 & Caustic potash & AR & ACROS \\
2 & Calcium hypochlorite & AR & ACROS \\
3 & Potassium nitrate & AR & ACROS \\
4 & Sodium sulfite & AR & ACROS \\
5 & Cyanoacrylate & AR & ACROS \\
6 & Butanol & AR & ACROS \\
7 & Glacial acetic acid & AR & ACROS \\
8 & Anhydrous ethanol & AR & ACROS \\
9 & Cupric nitrate & AR & ACROS \\
10 & Naphthalene & AR & ACROS \\
11 & Phenanthrene & AR & ACROS \\
12 & Pyrene & AR & ACROS \\
\hline
\end{tabular}

structure, aromatic hydrocarbon in oxidation might generate series of intermediates. There are some potential environmental risks; it is difficult to guarantee the safety of drinking water. This paper completes the transformation and removal of aromatic hydrocarbons in micropolluted water, in view of the refractory aromatic hydrocarbon in micropolluted source water and in view of potassium ferrate treatment technology.

\section{Experiment Methods}

2.1. Experiment Materials and Reagents. The main reagents and specifications used in the experiment are shown in Table 1.

2.2. Instruments and Equipment. The main instruments and related information used in the experiment are shown in Table 2. The infrared spectrum of potassium ferrate by Fourier infrared spectrometer (FTIR) is drawn, and the infrared characteristic peaks of potassium ferrate crystal are measured. Fluorescence spectrophotometer determines the degradation rate of the sample. Gas chromatographymass spectrometry instrument determines the intermediate of sample transformation and removal.

Some small instruments used in experiments consist of the beaker, $1000 \mathrm{~W}$ long arc xenon lamp, magnetic stirrer, electronic balance, separatory funnel, vacuum pump, the suction filter device, and so forth.

2.3. The Preparation of Potassium Ferrate. Proper $3 \mathrm{~mol} / \mathrm{L}$ and $13 \mathrm{~mol} / \mathrm{L}$ saturated potassium hydroxide solutions, cooling to room temperature, are prepared, respectively, for subsequent experiments. $15 \mathrm{~g}$ calcium hypochlorite is weighed in a small beaker; $25 \mathrm{~mL}$ of $13 \mathrm{~mol} / \mathrm{L}$ potassium hydroxide solution is added in slight agitation with a glass rod. The resulting solution is sucked with 800 -mesh filter cloth to leach impurities such as potassium chloride, collecting the yellowgreen filtrate. $20 \mathrm{~mL}$ cooling saturated potassium hydroxide solution is added with batch into the yellow-green filtrate. Impurities are removed by careful control of temperature, sufficient mixing, and air pump filtration, and then alkaline saturated potassium hypochlorite solution is obtained. $8 \mathrm{~g}$ $\mathrm{Fe}\left(\mathrm{NO}_{3}\right)_{3} \cdot 9 \mathrm{H}_{2} \mathrm{O}$ is weighed and is ground to powder and then is added slowly into the alkaline saturated potassium hypochlorite solution in batch with vigorous agitation. This reaction is exothermic with the ice-bath to control reaction temperature and stirring. The oxidation reaction is fast; soon the solution turns into purple. After 1-hour sufficient reaction, $90 \mathrm{~mL}$ cooling saturated potassium hydroxide solution was added into purple-black solution. The mixture is continuously stirred for $5 \mathrm{~min}$ and then is allowed to stand to cool in ice-bath for 30-40 min, followed by quickly sucking filtration with air pump. The liquor is abandoned and the resulting filtered cake, a crude potassium ferrate product, survives. The crude potassium ferrate product is washed 3 times with $3 \mathrm{~mol} / \mathrm{L}$ potassium hydroxide solution, $5 \mathrm{~mL}$ for each washing. The resulting potassium ferrate is redissolved, and filtration is done with air pump sucking. Filter cake is withdrawn, whereas the liquor is retained. The liquor is poured into distillation flask in ice-water bath, with addition of $40 \mathrm{~mL}$ cooling saturated potassium hydroxide solution. Recrystallization for 20 30 minutes is done, followed by sucking filtration with air pump. The resulting filter cake is the potassium ferrate, leached with $\mathrm{n}$-hexane $(4 \times 25 \mathrm{~mL})$, with pentane $(4 \times 25 \mathrm{~mL})$, with methanol $(4 \times 10 \mathrm{~mL})$, and with ether $(2 \times 10 \mathrm{~mL})$, respectively. The final product potassium ferrate crystal (purple-black), dried at $65^{\circ} \mathrm{C}$ for 2 hours, weighed, and kept in the drier, is prepared for subsequent use.

2.4. The Experiment of Potassium Ferrate to Remove Aromatic Hydrocarbon. Self-made potassium ferrate transforms aromatic hydrocarbon. In the micropolluted water medium $(\mathrm{pH}$ $=7.1$ ) with naphthalene, phenanthrene, and pyrene, the ability of potassium ferrate to transform aromatic hydrocarbons is explored on the basis of reaction time, potassium ferrate amounts, and removal of aromatic hydrocarbon.

On account of water quality of Chongqing Water Plant, polluted water by aromatic hydrocarbon is simulated by artificial addition of $200 \mu \mathrm{g} / \mathrm{L}$ aromatic hydrocarbon. The $\mathrm{pH}$ of the water is $7.1 .1000 \mathrm{~mL}$ of the polluted water is taken into a beaker with addition of different quantity of potassium ferrate, carrying out oxidation reaction with magnetic stirrer. At a specified time point $20 \mathrm{~mL}$ of the said solution is taken out of reaction system, followed by addition of sodium sulfite as termination agent. The content of aromatics remaining in the solution is determined by fluorescence spectrophotometer.

2.5. The Main Testing Index and Method. The removal of aromatic hydrocarbon is quantitatively determined by fluorescence spectrophotometer. The exciting wavelength and emission wavelength of three materials are as follows: naphthalene EX/EM is $218 / 332 \mathrm{~nm}$, phenanthrene EX/EM is $250 / 362 \mathrm{~nm}$, and pyrene EX/EM is $238 / 372 \mathrm{~nm}$, respectively. Excitation and emission slit are $5 \mathrm{~nm}$; scanning speed is $2400 \mathrm{~nm} / \mathrm{min}$

The properties of phenanthrene oxidation intermediate are determined by gas chromatography-mass spectrometry. After $30 \mathrm{~min}$ reaction to attain constant state, $200 \mathrm{~mL}$ solution is taken out from phenanthrene reaction system, followed 
TABLE 2: Main equipment and the respective specifications.

\begin{tabular}{|c|c|c|c|}
\hline Number & Instrument name & Instrument model & Manufacturer \\
\hline 1 & $\mathrm{X}$-ray diffractometer & $\mathrm{XD}-2$ & SHIMADZU, Japan \\
\hline 2 & UV spectrometer & UV-3010 & SHIMADZU, Japan \\
\hline 3 & Fourier transform infrared spectrometer & IRPrestige-21 & SHIMADZU, Japan \\
\hline 4 & Fluorescence spectrophotometer & F-7000 & SHIMADZU, Japan \\
\hline 5 & Gas chromatograph-mass spectrometer (GC-MS) & 6890N GC/5973 MS & SHIMADZU, Japan \\
\hline
\end{tabular}

by introduction into $400 \mathrm{~mL}$ separatory funnel. $6 \mathrm{~g} \mathrm{NaCl}$ is added into the filtered solution. When completely dissolved, $10 \mathrm{~mL} \mathrm{CH}_{2} \mathrm{Cl}_{2}$ is poured. The said solution is shaken for $10 \mathrm{~min}$, and then it was allowed to stand for $5 \mathrm{~min}$. $\mathrm{CH}_{2} \mathrm{Cl}_{2}$ layer is transferred to the pear-shaped flask. Additional $10 \mathrm{~mL}$ $\mathrm{CH}_{2} \mathrm{Cl}_{2}$ is put into the separative funnel twice, $5 \mathrm{~mL}$ for each. The solution was allowed to stand until organic layer survives. Then, the organic layer is merged and transferred. Anhydrous sodium sulfate is added into $\mathrm{CH}_{2} \mathrm{Cl}_{2}$ layer for drying. $\mathrm{CH}_{2} \mathrm{Cl}_{2}$ layer is concentrated at low temperature in the rotary evaporator; highly pure nitrogen is bubbled into the said layer until only $1 \mathrm{~mL}$ of the said solution is left. GCMS analysis is applied.

Gas chromatography-mass spectrometry, Agilent 6890 Net GC-Agilent 5973 Net MS series, is used; chromatographic column for HP-5 MS quartz capillary column $(25 \mathrm{~m} \times$ $0.25 \mathrm{~mm} \times 0.25 \mu \mathrm{m})$. The pattern of input sample is branchstream-free system, carrier gas is helium gas, and the flow rate is $1.0 \mathrm{~mL} / \mathrm{min}$. Temperature raising procedure is $40 \sim 290^{\circ} \mathrm{C}$ $\left(10^{\circ} \mathrm{C} / \mathrm{min}\right)$. Ionization mass spectrometry conditions are as follows: voltage is $70 \mathrm{eV}$ and the scanning range is of $30 \sim 400$ $\mathrm{m} / \mathrm{z}$.

Fourier transformed infrared spectrometer and ultraviolet-visible spectrometer are used to qualitatively characterize potassium ferrate.

\section{The Results and Discussion}

3.1. The Characterization of Potassium Ferrate Crystal. Fourier infrared spectrometer determined the purple crystal infrared spectrum at experimental conditions, as shown in Figure 1.

As can be seen in the figure, there is an absorption peak at $808 \mathrm{~cm}^{-1}$, which is potassium ferrate crystal $\mathrm{Fe}-\mathrm{O}$ bond stretching vibration characteristic peak. The peak is considered as the infrared characteristic peak of potassium ferrate. Other impurity peaks in the infrared spectrogram should be water vapor occurring in the instrument and the characteristics of absorption peak of the organic solvent used in the purification elution $[15,16]$.

Potassium Ferrate Ultraviolet-Visible Absorption Spectrum Characteristics. $0.0934 \mathrm{~g}$ purple crystal is weighed to prepare $100 \mathrm{~mL}$ of potassium ferrate solution in volumetric flask. Ultraviolet-visible spectrometer is used to test the ultravioletvisible absorption spectrum, as shown in Figure 2.

As can be seen in the figure, there is an obvious characteristic absorption peak at $510 \mathrm{~nm}$, which is ultraviolet-visible

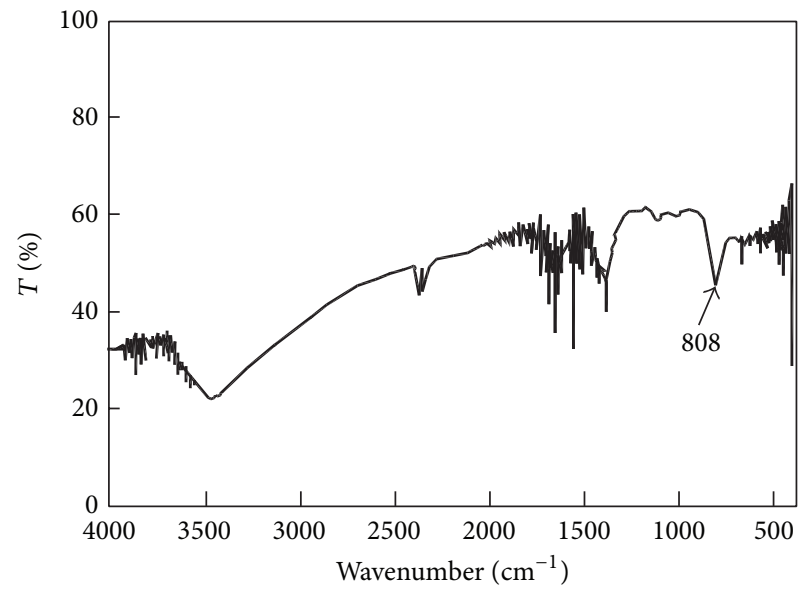

FIgURE 1: FTIR spectra of $\mathrm{K}_{2} \mathrm{FeO}_{4}$.

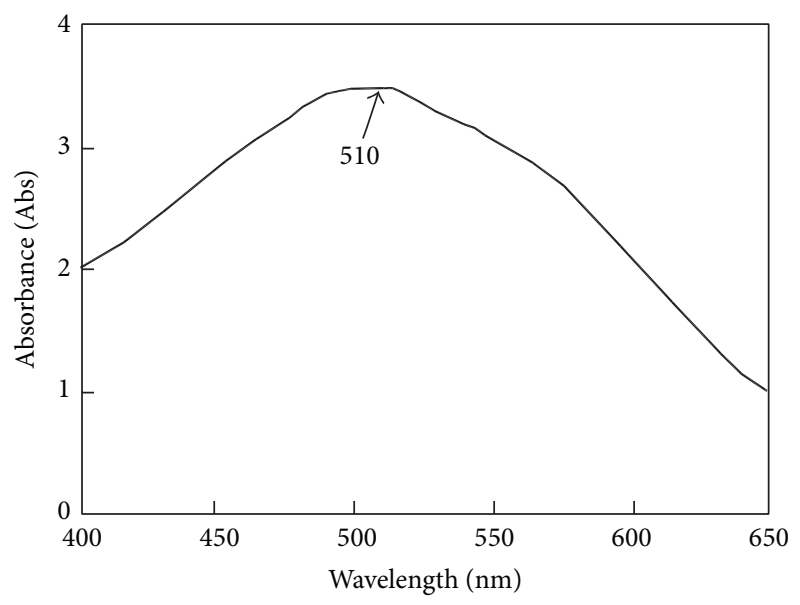

Figure 2: UV-Vis DRS of $\mathrm{K}_{2} \mathrm{FeO}_{4}$.

characteristic absorption peak of potassium ferrate. The peak is also considered as the quantitative analysis wavelength.

\subsection{The Influence of Reaction Time on Aromatic Hydrocarbon} Removal. When the oxidant concentration of potassium ferrate is at $6 \mathrm{mg} / \mathrm{L}$, the removal percentage of three kinds of aromatic hydrocarbon along with the change of time is shown in Figure 3. As can be seen in the figure, the transformation oxidation process of potassium ferrate for three kinds of aromatic hydrocarbon mainly occurred in the initial 5-10 minutes when the removal percentage of 


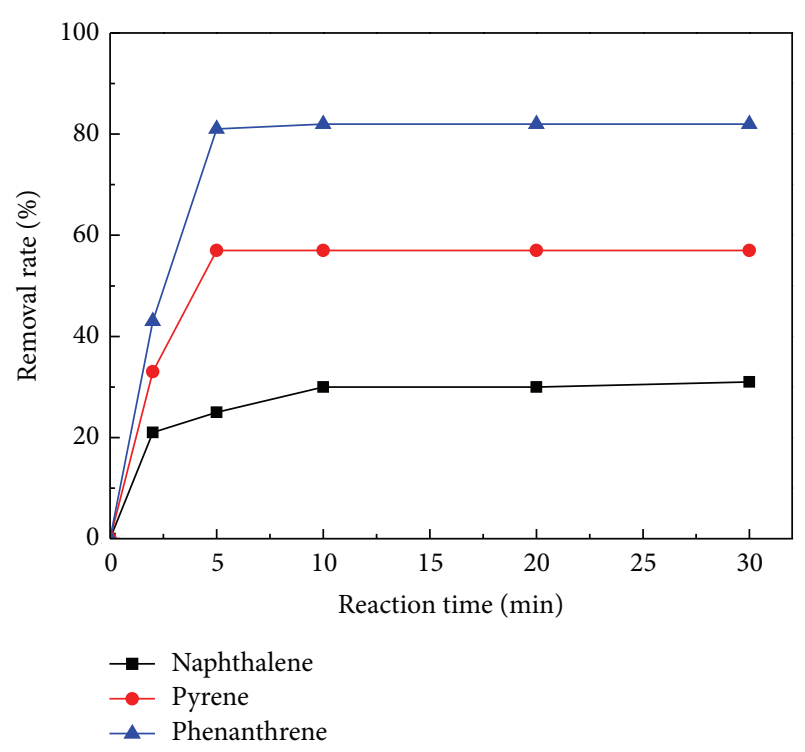

FIGURE 3: Influence of reaction time on aromatic hydrocarbons conversion and removal.

aromatic hydrocarbon increased rapidly as time increased. In five minutes later, the removal percentage of aromatic hydrocarbon only increased slightly. The result is consistent with the degradation rule of potassium ferrate oxidation degrading BPA [17] and arsenic [18]. Furthermore, it can be seen that over $80 \%$ final removal percentage of phenanthrene, 37\% that of naphthalene, and 58\% that of pyrene are developed, respectively. This shows that potassium ferrate can effectively transform phenanthrene in a relatively short time. These results are consistent with potassium ferrate high activity and instability. By comparison of three kinds of aromatic hydrocarbon removal percentage it is found that potassium ferrate has high oxidation, but to different samples its oxidation ability is not the same. Relatively, phenanthrene is most likely to be transformed, and naphthalene is more stable. The experiment results consisted of the three substances' delocalization. According to the experimental results, the oxidation reaction time of potassium ferrate to the three substances is preliminarily identified to be $20 \mathrm{~min}$.

3.3. The Influence of the Amount on Aromatic Hydrocarbon Removal Effect. The influence of potassium ferrate amount on aromatic hydrocarbon removal ability is shown in Figure 4, derived from measurement of samples in $20 \mathrm{~min}$. As can be seen in Figure 4, as potassium ferrate amount increases, three kinds of aromatic hydrocarbon removal percentage gradually increased. Relatively, at the same reagent amount, phenanthrene has the highest removal percentage. When the potassium ferrate concentration is $10 \mathrm{mg} / \mathrm{L}$, phenanthrene has the highest removal percentage, reaching $98 \%$; pyrene and naphthalene were $84 \%$ and $61 \%$, respectively. Further improvement of amount of oxidant potassium ferrate cannot obviously improve the removal percentage for the three substances. Removal of the aromatic hydrocarbons in micropolluted source water, therefore, has the most appropriate $10 \mathrm{mg} / \mathrm{L}$ potassium ferrate amount.

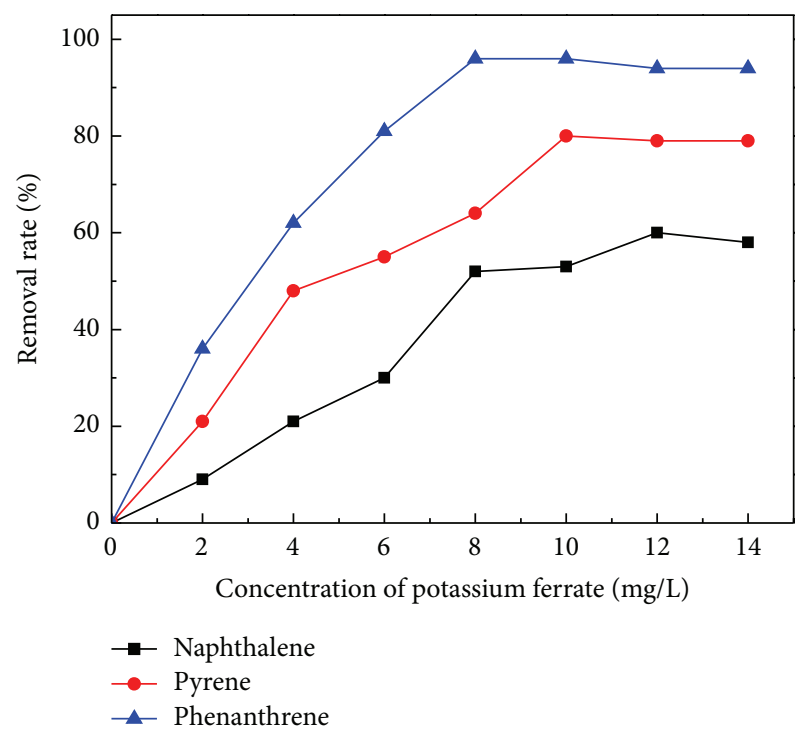

FIGURE 4: Influence of $\mathrm{K}_{2} \mathrm{FeO}_{4}$ concentration on aromatic hydrocarbons conversion and removal.

Potassium ferrate has strong oxidation, regardless of being in acidic medium or alkaline medium. The electrode potential is significantly higher than potassium permanganate and potassium dichromate; it can oxidize most organic substances. In hydrolysis process potassium ferrate produces strong oxidation hydroxyl free radicals [19]; at the same time, the unstable intermediate pentavalent iron and tetravalent iron also have a strong oxidizing ability [20]. The final reduction product is ferric iron. Potassium ferrate removal of aromatic hydrocarbon is the outcome of concerted action of these strong oxidizing substances. Low concentration potassium ferrate can transform removal of aromatic hydrocarbon. When the reaction time is $20 \mathrm{~min}$, potassium ferrate concentrations are 12,8 , and $10 \mathrm{mg} / \mathrm{L}$; naphthalene, phenanthrene, and pyrene removal percentage reached the maximum value, that is, $67 \%, 98 \%$, and $84 \%$, respectively.

3.4. Analysis of the Intermediate of Removing Phenanthrene. Due to the special stability of benzene ring structure, intermediate products can be generated during the oxidation process of aromatic substances. Thus, the removal process of aromatic substances has a potential environmental risk. Therefore, we studied the concrete structure of intermediate by GC-MS analysis.

Following are the GC of potassium ferrate system and mass spectrogram of some intermediates to transform phenanthrene.

Figure 5 is the GC of potassium ferrate system to transform phenanthrene.

As can be seen in the figure, the retention time of phenanthrene and main potassium ferrate reaction products was $12.443,13.375,13.575$, and $18.139 \mathrm{~min}$, respectively. In terms of corresponding mass spectrum, the peak retention time at $12.453 \mathrm{~min}$ is the undegraded phenanthrene peak. Compared 


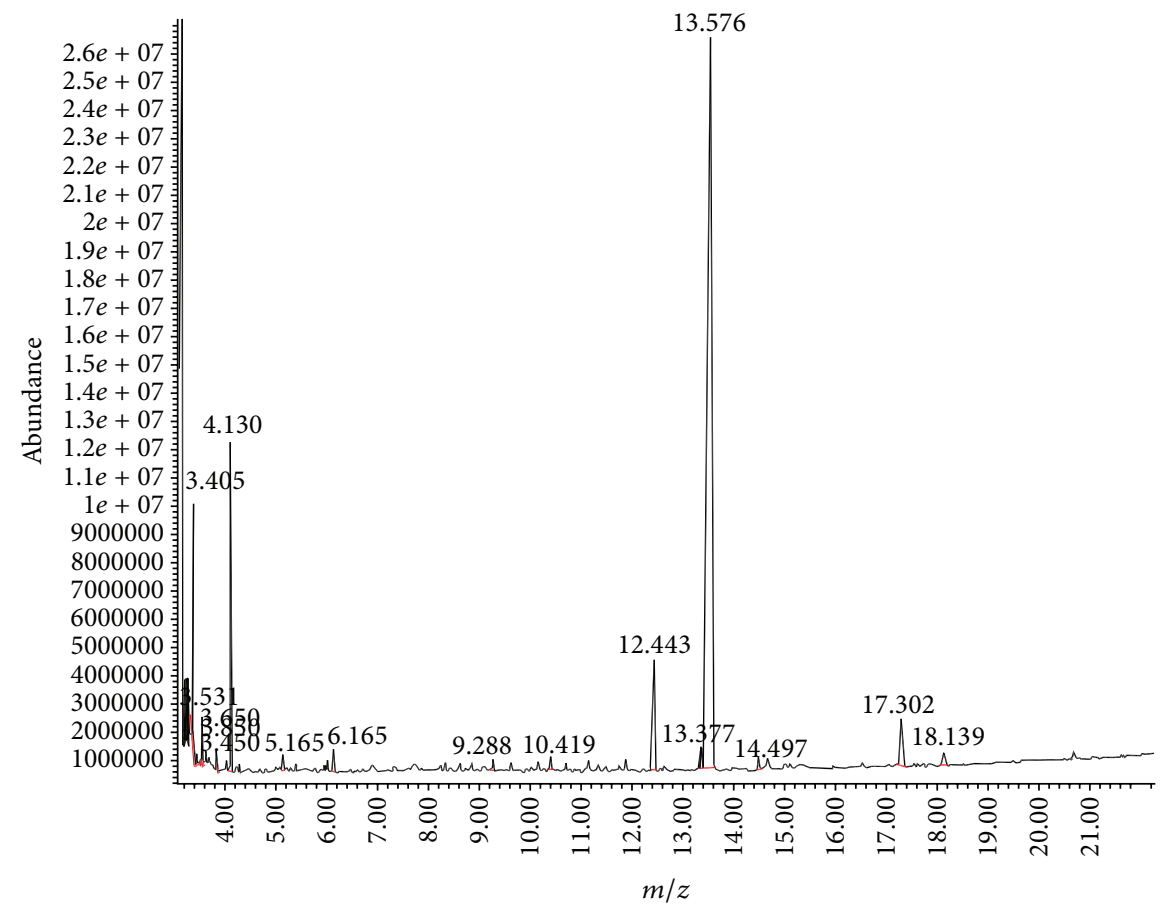

FIGURE 5: The GC map of phenanthrene conversion and removal by potassium ferrate system.

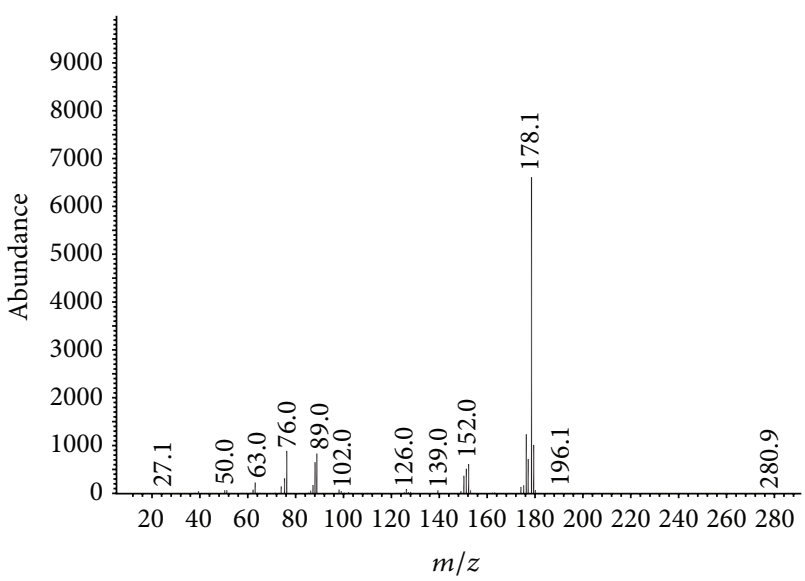

FIGURE 6: The mass spectrogram of $12.443 \mathrm{~min}$.

with mass spectrometry retrieve library standard spectrogram, other intermediates mainly include butyl phthalate, 9,10-phenanthraquinone, and diphenyl-2,2' -biformaldehyde. In addition, there are some significant intermediates, such as 9-fluorenone and dimethylbenzenal. And the peak of the main production prepared by phenanthrene reaction with potassium ferrate is at $13.575 \mathrm{~min}$; the component area percentage accounted for $82.66 \%$. Specific mass spectrum and structural formula of main products are as follows.

(1) The mass spectrum peak diagram in GC of retention time at $12.443 \mathrm{~min}$ is as shown in Figure 6.

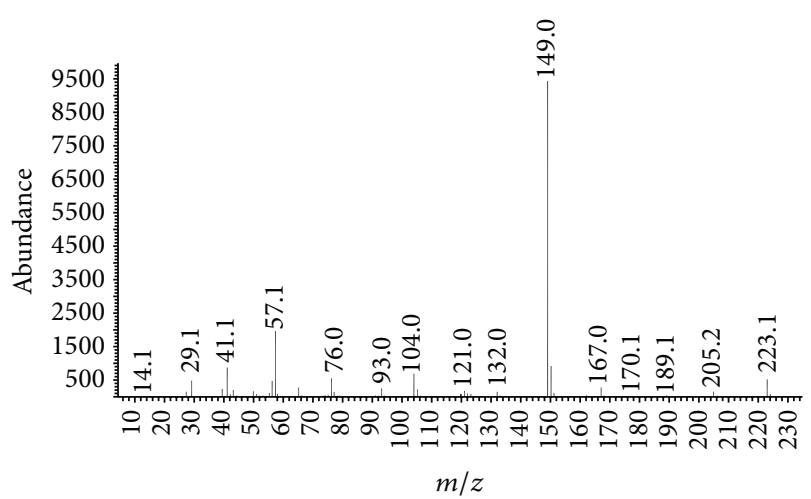

FIgURE 7: The mass spectrogram of $13.375 \mathrm{~min}$.

According to comparison with the spectral library spectrum standard substance, phenanthrene residue is identified in original solution, with $96 \%$ matching degree with the literatures, as the following structural formula:

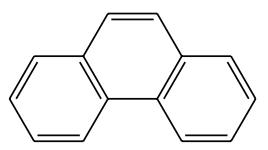

(2) The peak mass spectrum diagram in GC as of retention time at $13.375 \mathrm{~min}$ is as shown in Figure 7.

According to comparison with the spectral library spectrum standard substance, this material is identified as butyl 
phthalate, with $86 \%$ matching degree, and its formula is as follows:<smiles>CC(C)COC(=O)c1ccccc1C(=O)OCC(C)C</smiles>

This material area percentage is only $1.32 \%$, to show that this material accounts for a very small proportion in the product. This kind of material can be the intermediate produced by complex reaction of oxidation.

(3) The peak mass spectrum diagram in GC of retention time at $13.575 \mathrm{~min}$ is as shown in Figure 8.

According to comparison with the spectral library spectrum standard substance, this material is identified as 9,10phenanthraquinone, with $92 \%$ matching degree, and its formula is as follows:

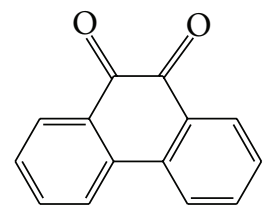

This material area percentage is $82.66 \%$, to show that this material accounts for a very high proportion in the product. It is the main product of potassium ferrate in transformation of phenanthrene. Phenanthrene is made up of three benzene rings, where 9,10-position has strong chemical reactivity, prone to oxidation. In potassium ferrate oxidation system, phenanthrene gives Fe (VI) electron to be oxidized. In addition, phenanthrene can get $98 \%$ transformation in ferrate oxidation system, but it cannot completely inorganic, but is turned into 9,10-phenanthrenequinone. According to the research results by Lawton et al. [21], quinone structure is a kind of functional group more biophile than benzene ring. Its relatively weak biological resistance is advantageous over the biochemical conversion process. It changes aromatic hydrocarbon environmental durability characteristics and, to some extent, reduces the environmental risk.

(4) The peak mass spectrum diagram in GC of retention time at $18.139 \mathrm{~min}$ is as shown in Figure 9.

According to comparison with the spectral library spectrum standard substance, this material is identified as diphenyl$2,2^{\prime}$-biformaldehyde, with $70 \%$ matching degree, and its formula is as follows:<smiles>O=Cc1ccccc1-c1ccccc1C=O</smiles>

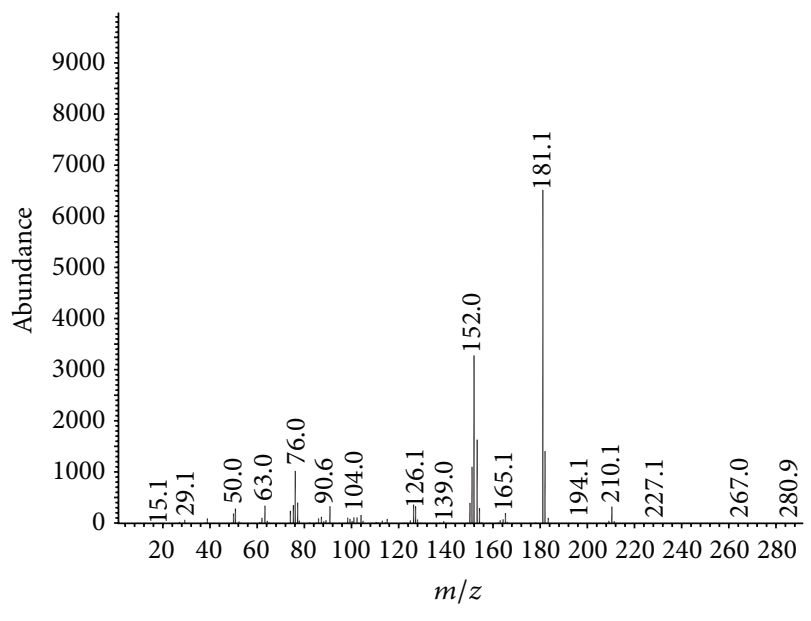

FIGURE 8: The mass spectrogram of $13.575 \mathrm{~min}$.

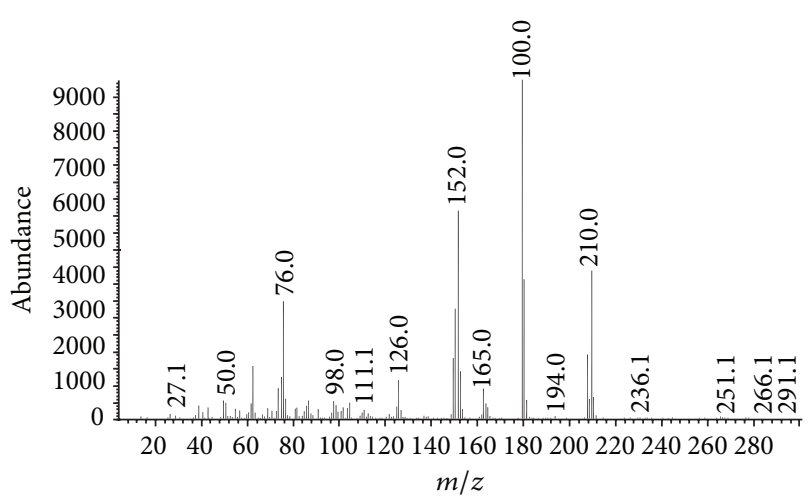

FIGURE 9: The mass spectrogram of $18.139 \mathrm{~min}$.

This material area percentage is only $1.05 \%$, showing that this material accounts for a very small proportion in the product. In the process of potassium ferrate oxidation of phenanthrene, Fe (VI) to be reduced generates Fe (V), Fe (IV), and $\mathrm{Fe}$ (III) $[22,23]$ through single electron transfer steps. Phenanthrene 9,10-position has strong chemical reactivity, prone to oxidation. In potassium ferrate oxidation system, phenanthrene gives Fe (VI) electron to be oxidized. Since this is an oxidation system, diphenyl-2,2' -biformaldehyde is easy to be oxidized by potassium ferrate, so its proportion in products accounted for quite a few.

3.5. Primary Analysis of Oxidation System Mechanism to Transform Phenanthrene. In the process of potassium ferrate to oxidize phenanthrene, $\mathrm{Fe}(\mathrm{VI})$ to be reduced generates $\mathrm{Fe}$ (V), Fe (IV), and Fe (III) [24, 25] through single electron transfer steps. Phenanthrene is made up of three benzene rings, of which 9,10-position has strong chemical reactivity prone to oxidation. In potassium ferrate oxidation system, phenanthrene gives Fe (VI) electron to be oxidized. According to the single electron transfer mechanism, the possible reaction of potassium ferrate and phenanthrene is shown in Figure 10. 


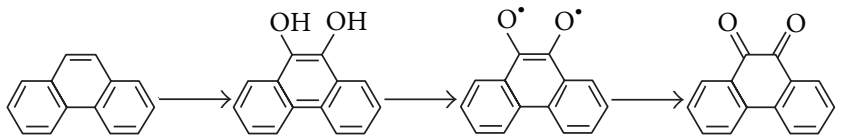

FIGURE 10: The possible way of reaction of potassium ferrate with phenanthrene.

\section{Conclusions}

This experiment adopts the hypochlorite oxidation approach to constantly synthesize potassium ferrate. The transformation oxidation process of potassium ferrate for aromatic hydrocarbons mainly occurred in the initial 5-10 minutes. Compared with aromatic hydrocarbon transformation removal percentage, it was found that potassium ferrate has high oxidation, but to different samples its transformation oxidation ability is not the same. Relatively, phenanthrene is most likely to be transformed, and naphthalene is more stable. In potassium ferrate, to remove phenanthrene, in GC and in the intermediate mass spectrum analysis, phenanthrene gives electron to $\mathrm{Fe}(\mathrm{VI})$ of potassium ferrate oxidation system, so that phenanthrene oxidation occurs. Thus possible reaction path is proposed: the quinone structure is a kind of functional group more biophile than benzene ring. Because of its biologically weak resistance, it is advantageous to the biochemical conversion process, to conversion of aromatic hydrocarbon's environmental durability characteristics, and, to some extent, to reduction of the environmental risk.

\section{Conflict of Interests}

The authors declare that there is no conflict of interests regarding the publication of this paper.

\section{References}

[1] N. F. Y. Tam, L. Ke, X. H. Wang, and Y. S. Wong, "Contamination of polycyclic aromatic hydrocarbons in surface sediments of mangrove swamps," Environmental Pollution, vol. 114, no. 2, pp. 255-263, 2001.

[2] M. Sanders, S. Sivertsen, and G. Scott, "Origin and distribution of polycyclic aromatic hydrocarbons in surficial sediments from the Savannah River," Archives of Environmental Contamination and Toxicology, vol. 43, no. 4, pp. 438-448, 2002.

[3] R.-A. Doong and Y.-T. Lin, "Characterization and distribution of polycyclic aromatic hydrocarbon contaminations in surface sediment and water from Gao-ping River, Taiwan," Water Research, vol. 38, no. 7, pp. 1733-1744, 2004.

[4] F. Lin, H. Ye, and Y. Song, "Preparation and electrochemical properties of $\mathrm{Li}$-doped $\mathrm{BaFeO}_{4}$ as electrode material for lithium-ion batteries," Electrochemistry Communications, vol. 2, no. 6, pp. 531-534, 2001.

[5] V. K. Sharma, "Potassium ferrate(VI): an environmentally friendly oxidant," Advances in Environmental Research, vol. 6, no. 2, pp. 143-156, 2002.

[6] M. Alsheyab, J.-Q. Jiang, and C. Stanford, "On-line production of ferrate with an electrochemical method and its potential application for wastewater treatment-a review," Journal of Environmental Management, vol. 90, no. 3, pp. 1350-1356, 2009.
[7] J. Q. Jiang, S. Wang, and A. Panagoulopoulos, “The exploration of potassium ferrate(VI) as a disinfectant/coagulant in water and wastewater treatment," Chemosphere, vol. 63, no. 2, pp. $212-$ 219, 2006.

[8] Y. Y. Eng, V. K. Sharma, and A. K. Ray, "Ferrate(VI): green chemistry oxidant for degradation of cationic surfactant," Chemosphere, vol. 63, no. 10, pp. 1785-1790, 2006.

[9] B. L. Yuan, Y. B. Li, X. D. Huang, H. Liu, and J. Qu, "Fe(VI)assisted photocatalytic degradating of microcystin-LR using titanium dioxide," Journal of Photochemistry and Photobiology A: Chemistry, vol. 178, no. 1, pp. 106-111, 2006.

[10] D. Tiwari, H.-U. Kim, B.-J. Choi et al., "Ferrate(VI): a green chemical for the oxidation of cyanide in aqueous/waste solutions," Journal of Environmental Science and Health A: Toxic/Hazardous Substances \& Environmental Engineering, vol. 42, no. 6, pp. 803-810, 2007.

[11] V. K. Sharma, "Use of iron(VI) and iron(V) in water and wastewater treatment," Water Science and Technology, vol. 49, no. 4, pp. 69-74, 2004.

[12] J. Q. Jiang, "Research progress in the use of ferrate(VI) for the environmental remediation," Journal of Hazardous Materials, vol. 146, no. 3, pp. 617-623, 2007.

[13] C. Li, Z. X. Li, N. Graham et al., "The aqueous degradation of bisphenol A and steroid estrogens by ferrate," Water Research, vol. 42, no. 1-2, pp. 109-120, 2008.

[14] V. K. Sharma, S. K. Misra, and A. K. Ray, "Kinetic assessment of the potassium ferrate(VI) oxidation of antibacterial drug sulfamethoxazole," Chemosphere, vol. 62, no. 1, pp. 128-134, 2006.

[15] F. Dong, Y. Sun, M. Fu, Z. Wu, and S. C. Lee, "Room temperature synthesis and highly enhanced visible light photocatalytic activity of porous $\mathrm{BiOI} / \mathrm{BiOCl}$ composites nanoplates microflowers," Journal of Hazardous Materials, vol. 219-220, pp. 26-34, 2012.

[16] F. Dong, Y. Sun, M. Fu, W.-K. Ho, S. C. Lee, and Z. Wu, "Novel in situ $\mathrm{N}$-doped $(\mathrm{BiO})_{2} \mathrm{CO}_{3}$ hierarchical microspheres self-assembled by nanosheets as efficient and durable visible light driven photocatalyst," Langmuir, vol. 28, no. 1, pp. 766-773, 2012.

[17] X. L. Zhu and C. W. Yuan, "Photocatalytic degradation of pesticide pyridaben on $\mathrm{TiO}_{2}$ particles," Journal of Molecular, vol. 229, no. 1-2, pp. 95-105, 2005.

[18] M. A. Rahman and M. Qamar, "Semiconductor mediated photocatalysed degradation of a pesticide derivative, acephate in aqueous suspensions of titanium dioxide," Journal of Advanced Oxidation Technologies, vol. 9, no. 1, pp. 103-109, 2006.

[19] M. Iwasaki, M. Hara, H. Kawada et al., "State of arts for bleach plant effluent closure," Journal of Colloid and Interface Scienee, vol. 224, no. 1, pp. 202-204, 2000.

[20] D. C. Hurum, A. G. Agrios, K. A. Gray, T. Rajh, and M. C. Thurnauer, "Explaining the enhanced photocatalytic activity of Degussa P25 mixed-phase $\mathrm{TiO}_{2}$ using EPR," Journal of Physical Chemistry B, vol. 107, no. 19, pp. 4545-4549, 2003.

[21] L. A. Lawton, P. K. J. Robertson, B. J. P. A. Cornish, I. L. Marr, and M. Jaspars, "Processes influencing surface interaction and photocatalytic destruction of microcystins on titanium dioxide photocatalysts," Journal of Catalysis, vol. 213, no. 1, pp. 109-113, 2003.

[22] S. Malato, J. Blanco, A. Vidal, and C. Richter, "Photocatalysis with solar energy at a pilot-plant scale: an overview," Applied Catalysis B: Environmental, vol. 37, no. 1, pp. 1-15, 2002. 
[23] A. G. Rincón, C. Pulgarin, N. Adler, and P. Peringer, "Interaction between E. coli inactivation and DBP-precursorsdihydroxybenzene isomers-in the photocatalytic process of drinking-water disinfection with $\mathrm{TiO}_{2}$," Journal of Photochemistry and Photobiology A Chemistry, vol. 139, no. 2-3, pp. 233241, 2001.

[24] W. He, J. Wang, H. Shao, J. Zhang, and C.-N. Cao, "Novel $\mathrm{KOH}$ electrolyte for one-step electrochemical synthesis of high purity solid $\mathrm{K}_{2} \mathrm{FeO}_{4}$ : comparison with $\mathrm{NaOH}$,' Electrochemistry Communications, vol. 7, no. 6, pp. 607-611, 2005.

[25] B.-L. Yuan, X.-Z. Li, and N. Graham, "Aqueous oxidation of dimethyl phthalate in a $\mathrm{Fe}(\mathrm{VI})-\mathrm{TiO}_{2}-\mathrm{UV}$ reaction system," Water Research, vol. 42, no. 6-7, pp. 1413-1420, 2008. 

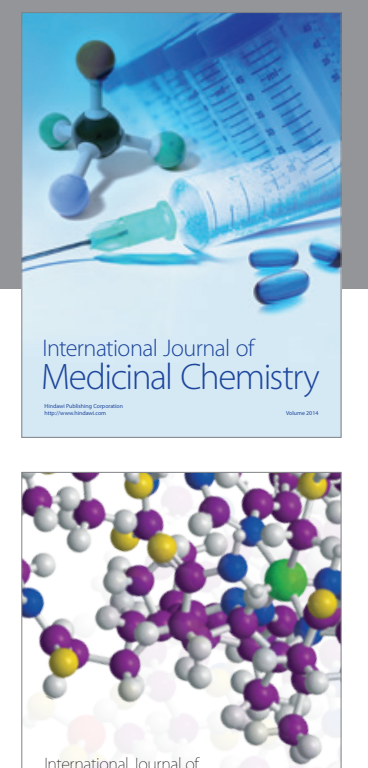

\section{Carbohydrate} Chemistry

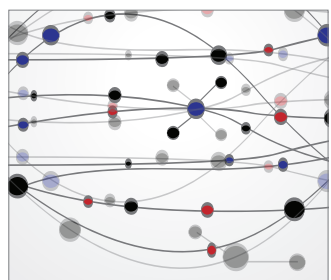

The Scientific World Journal
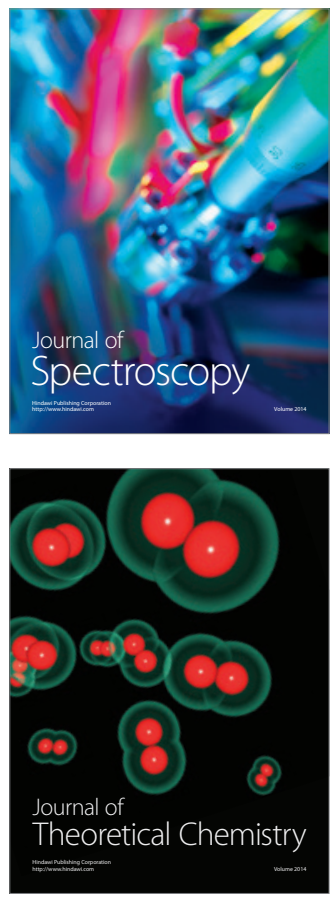
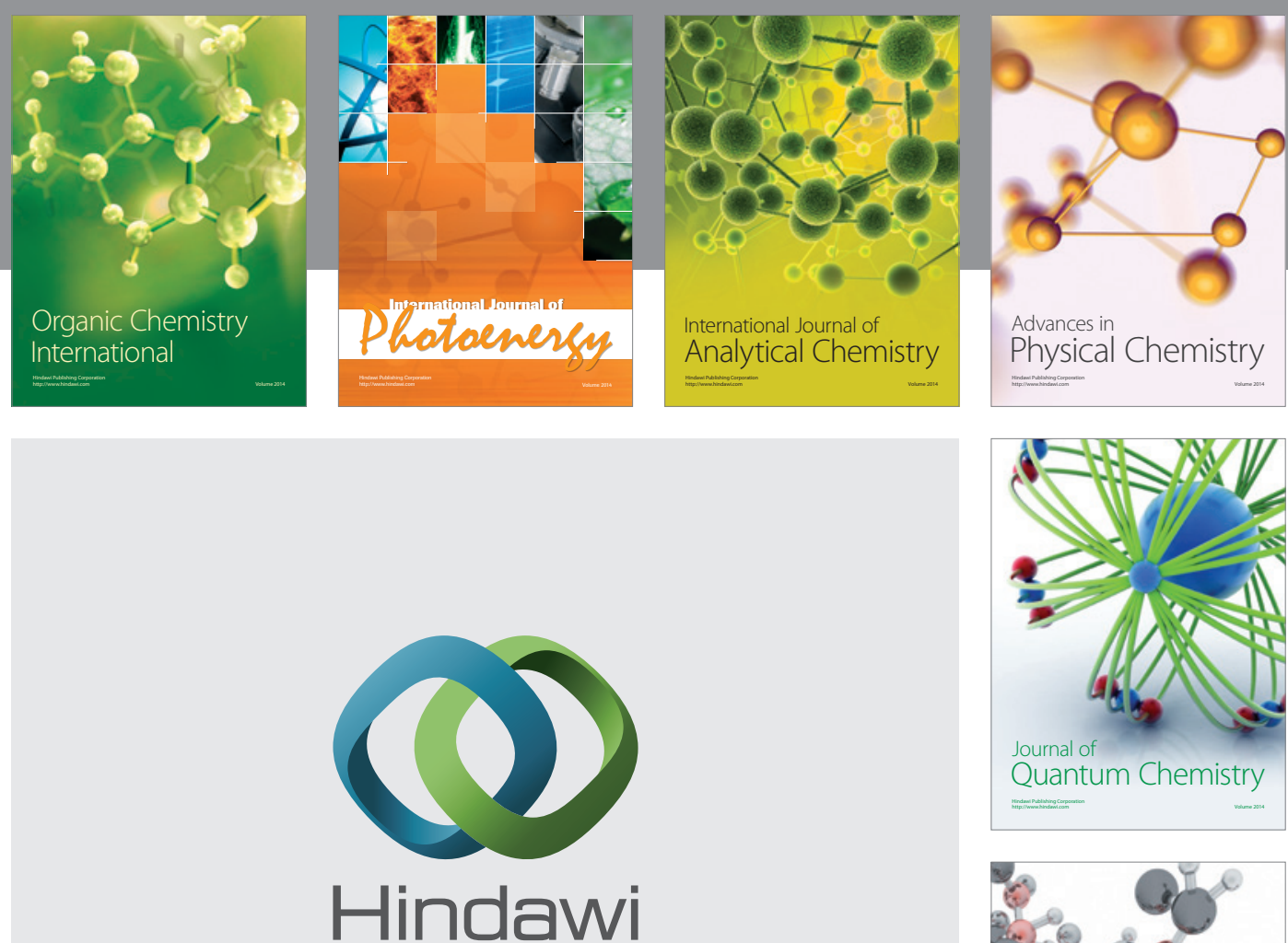

Submit your manuscripts at

http://www.hindawi.com

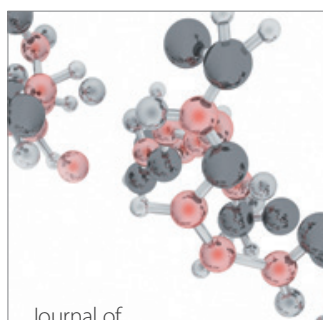

Analytical Methods

in Chemistry

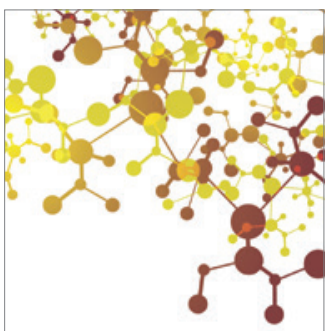

Journal of

Applied Chemistry

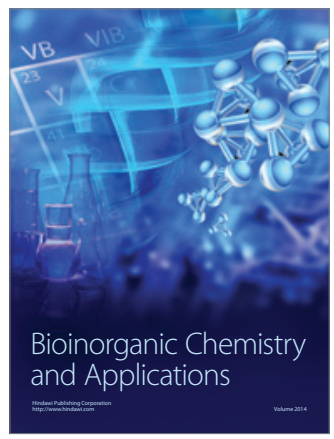

Inorganic Chemistry
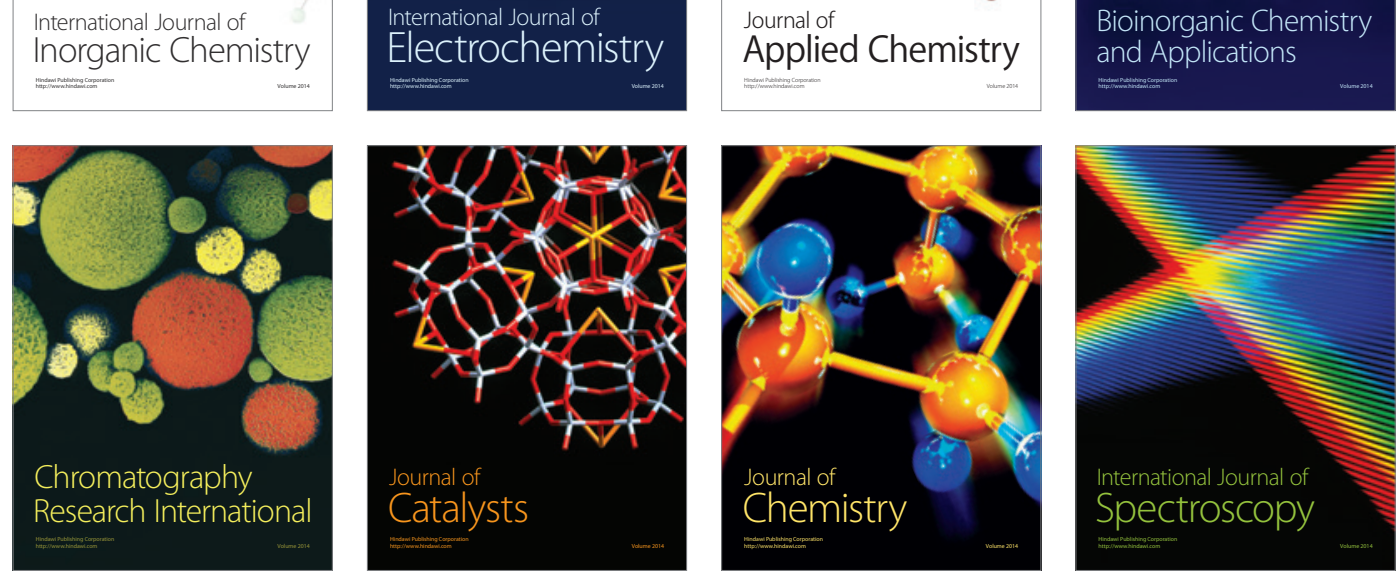